\title{
CARACTERIZAÇ̃̃O ESTRUTURAL DO COMPÓSITO PANI-MAGNETITA COM POTENCIAL APLICABILIDADE EM SENSOR ELETROQUÍMICO
}

\author{
Caroline Zanotto $^{\mathrm{a}}$, Patrícia T. Marques ${ }^{\mathrm{b}}$ e Eryza G. de Castro ${ }^{\mathrm{a}, *,(1)}$ \\ aDepartamento de Química, Universidade Estadual do Centro-Oeste, Campus CEDETEG, 85040-080 Guarapuava - PR, Brasil \\ bDepartamento de Química, Universidade Tecnológica Federal do Paraná, 85503-390 Pato Branco - PR, Brasil
}

Recebido em 02/12/2020; aceito em 23/04/2021; publicado na web em 19/05/2021

\begin{abstract}
STRUCTURAL CHARACTERIZATION OF THE PANI-MAGNETITE COMPOSITE WITH POTENTIAL APPLICABILITY IN ELECTROCHEMICAL SESNOR. This study presents the production of a composite formed between polyaniline and magnetite (Pani-Mag) through an easy and more economical synthetic route, which satisfactorily contemplates the principles of green chemistry. The production of new materials for technological application also needs to be focused on less waste generation, and greater sustainability. FTIR and DRX analyzes confirm that the interactions between the polymeric phases and the oxide particles occurred in a synergistic way. Saturation magnetization has a value of $33.5 \mathrm{emu} \mathrm{g}^{-1}$, being compatible with reference values in the literature for Pani-Mag composites. The electrochemical tests indicate an electrochemically stable material with the potential to be applied as an electrochemical sensor in the determination of ascorbic acid in solution.
\end{abstract}

Keywords: composites; Pani-magnetite; green chemistry; ascorbic acid.

\section{INTRODUÇÃO}

O crescente desenvolvimento dos polímeros condutores fez surgir os materiais compósitos multifuncionais, tornando-se promissor na área de química de materiais, devido à vasta possibilidade de aplicações tecnológicas, principalmente na área de dispositivos eletrônicos e eletroquímicos como sensores/biosensores, baterias, fotovoltaicos. ${ }^{1,2}$

Dentre os polímeros condutores, a Polianilina (Pani) merece destaque devido as propriedades de condutividade elétrica, baixo custo de obtenção, estabilidade em condições ambientes, ${ }^{3}$ além de facilidade de formar compósitos por rotas diversificadas de síntese, bom rendimento e dopagem simples por ácidos protônicos. ${ }^{1,4}$

No entanto, a Pani obtida por rotas químicas ou eletroquímicas tem solubilidade e processabilidade limitada, além de propriedades mecânicas ruins. Na tentativa de melhorar essas condições, compósitos foram produzidos com o intuito de unir em um único material e melhorar, as propriedades mecânicas, físicas, ópticas e condutoras de um polímero com as propriedades magnéticas de partículas de magnetita (Mag). De acordo com a literatura, esse sinergismo melhora as características e desempenho para aplicações em novos materiais. ${ }^{1,4-6}$

Como exemplos de estudos de aplicações para o compósito Pani-Mag, cita-se baterias de armazenamento, ${ }^{7}$ capacitores,${ }^{8}$ catálise ambientalmente sustentável, ${ }^{9}$ sensores de glicose com eletrodo modificado com Pani- $\mathrm{Fe}_{3} \mathrm{O}_{4}$-grafeno, ${ }^{10}$ sensores de etanol e colesterol usando magnetita como material de suporte para eletrodo. ${ }^{11}$

Esses estudos da literatura utilizam sínteses complexas, com excesso de reagentes, solventes orgânicos e substâncias auxiliares que apresentam risco potencial para o meio ambiente, saúde e de acidentes em laboratórios, além de equipamentos específicos. Pensando em sustentabilidade, esse estudo traz uma proposta de rota sintética simples, com mais praticidade, evitando desperdícios e consequentemente menor custo de obtenção.

Uma ferramenta aliada na etapa de síntese, com foco na sustentabilidade, é a utilização da métrica de análise da química verde,

*e-mail: eryza.castro@gmail.com como metodologia para avaliar quais parâmetros são satisfatórios para os princípios sustentáveis, e quais precisam ser melhorados.

Esse trabalho tem como objetivo sintetizar o compósito de Pani com partículas de magnetita, através de método de síntese simples, na perspectiva sustentável da química verde. A estrutura do compósito foi avaliada por caracterizações espectroscópicas, estruturais, magnéticas e eletroquímicas com intuito de analisar potencial de aplicabilidade como sensor eletroquímico para molécula de ácido ascórbico.

A molécula de ácido ascórbico (AA) foi escolhida nesse estudo por ser uma vitamina importante para sistemas bioquímicos e farmacológicos. Sabe-se que a deficiência de Vitamina $\mathrm{C}$ pode causar doenças, e seus níveis adequados atuam na prevenção de doenças respiratórias, cardiovasculares entre outras. ${ }^{12} \mathrm{O}$ ácido ascórbico é utilizado também na indústria alimentícia como potente antioxidante para prevenir mudanças indesejadas na cor e sabor de alimentos, mantendo a conservação. ${ }^{13}$

Diante da sua elevada importância, é essencial a utilização de métodos eficazes e alternativos para determinação do ácido ascórbico, como é o caso dos sensores, que através dos testes eletroquímicos pode determinar com precisão a concentração do ácido em amostras diversas, sendo possível detectar limites distintos em quantidades pequenas. Essa caracterização é importante para controle de qualidade na indústria alimentícia e em fórmulas farmacêuticas, assegurando quantidades recomendadas e adequadas de controle, evitando utilização em excesso.

\section{PARTE EXPERIMENTAL}

\section{Preparo do compósito}

As partículas de magnetita foram sintetizadas, partindo-se da metodologia descrita, ${ }^{8,14}$ na qual a mistura de cloretos de ferro $\left(\mathrm{FeCl}_{2}\right.$ $1,0 \mathrm{~mol} \mathrm{~L}^{-1} \mathrm{e} \mathrm{FeCl}_{3} 1,5 \mathrm{~mol} \mathrm{~L}^{-1}, 25 \mathrm{~mL}$ ) foi gotejada lentamente em uma solução de $50 \mathrm{~mL}$ de $\mathrm{NH}_{4} \mathrm{OH}(25 \%$ v/v), sob agitação vigorosa. O precipitado de coloração preta foi rapidamente formado e o sistema mantido em agitação por $2 \mathrm{~h}$. Em seguida o precipitado foi lavado com água destilada, até incolor, sendo decantado com auxílio de um imã de Neodímio (Neodímio, Ferro, Boro) de bloco (40x40x1 cm). 
O compósito Pani-Mag foi sintetizado a partir da metodologia adaptada, ${ }^{15,16} \mathrm{em}$ que as partículas de magnetita previamente sintetizadas $(0,5 \mathrm{~g})$ são dispersas em solução aquosa de DBSA (ácido dodecilbenzenosulfônico) (10 mL em $100 \mathrm{~mL}$ de água) por ultrassom durante 30 minutos. Após agitação mecânica $(800 \mathrm{rpm})$ constante por $1 \mathrm{~h}$, adicionou-se $1 \mathrm{~mL}$ do monômero anilina purificado, seguido de $5 \mathrm{~mL}$ do agente oxidante $\mathrm{FeCl}_{3}, 1 \mathrm{~mol} \mathrm{~L}{ }^{-1}$, mantendo agitação por mais $4 \mathrm{~h}$. O material foi lavado, centrifugado e seco para as análises posteriores. Um padrão de Pani sem inserção de partículas de magnetita foi sintetizado seguindo a mesma metodologia.

\section{Caracterizações}

Tanto o compósito quanto os precursores sintetizados foram caracterizados pelas mesmas técnicas descritas a seguir, como parâmetro de comparação.

Os espectros de FTIR foram obtidos na faixa de $400-4000 \mathrm{~cm}^{-1}$ em um espectrômetro Shimadzu Prestige-21 com as amostras em pastilhas de $\mathrm{KBr}$ sólido. Difratometria de raios X (DRX), foi realizada em um equipamento Rigaku Miniflex 600, tensão de $40 \mathrm{kV}$ e corrente de $15 \mathrm{~mA}$, em taxa de varredura $2^{\circ} \mathrm{min}^{-1}$, modo $2 \theta$ e intervalo de 10 a 60 graus.

Caracterização magnética de curva de histerese foi realizada em magnetômetro de amostra vibrante VMS (vibrating sample magnetometer), que foi calibrado nos eixos $\mathrm{x}, \mathrm{y}, \mathrm{z}$ para obter a resposta máxima do campo magnético da amostra. Para todas as medidas utilizou-se $25 \mathrm{mg}$ de amostra a temperatura ambiente, a variação de campo foi de -20 kOe até $20 \mathrm{kOe}$ com sensibilidade de $20 \mathrm{mV}$.

Caracterização eletroquímica pela técnica de Voltametria Cíclica (VC) realizada em equipamento EmStat da PalmSens, conectado ao notebook ou celular através do software PS Trace 5.5, sendo as medidas eletroquímicas realizadas em célula eletroquímica de 3 eletrodos. O eletrodo de trabalho foi de carbono vítreo modificado com as amostras (área da superfície $0,070 \mathrm{~cm}^{2}$ ). Para referência, utilizou-se o eletrodo de $\mathrm{Ag} / \mathrm{AgCl} / \mathrm{KCl} 3 \mathrm{~mol} \mathrm{~L}^{-1}$, e como contra eletrodo uma placa de platina de $1 \mathrm{~cm} \times 1 \mathrm{~cm}$. O preparo do eletrodo de trabalho consistiu em uma suspensão do material a ser analisado, com $1 \mathrm{~mL}$ de acetona (50\% v/v) gotejado sobre a superfície do mesmo. Após secagem prévia, uma gota de Nafion $(10 \% \mathrm{v} / \mathrm{v})$ foi necessária para melhor adesão da amostra na superfície do eletrodo. ${ }^{12}$

\section{Métrica da química verde}

Pensando em realizar sínteses de compósitos de maneira mais sustentável, econômica, com menor geração de resíduos, foram utilizados os princípios da química verde e construção da estrela verde para analisar numa perspectiva de índice de verdura. A métrica da química verde seguiu 10 princípios da construção da estrela verde (EV), que mediante critérios pré-definidos e planilhas preenchidas, obteve-se um padrão de verdura para o experimento. Para cada princípio são atribuídos valores 1, 2 ou 3, de acordo com informações pré analisadas em fichas de segurança de produtos químicos para cada reagente utilizado, produtos formados e resíduos, sendo que 3 é o máximo de verde que se deseja obter para o determinado parâmetro. Construiu-se a estrela, em que é possível obter a porcentagem de área verde em relação à área total, que se denomina IPE (índice de preenchimento máximo da estrela). ${ }^{17,18}$

\section{RESULTADOS E DISCUSSÃO}

\section{Caracterizações espectroscópias e estruturais}

A Figura 1 apresenta os difratogramas dos materiais produzidos. Para a magnetita, obteve-se os picos característicos da fase espinélio em 20: $18^{\circ}(111), 30^{\circ}(220), 35^{\circ}(311), 43^{\circ}(400), 53^{\circ}(422), 57^{\circ}(511)$, de acordo com Carta Cristalográfica JCPDS $190629,{ }^{19}$ confirmando a presença de $\mathrm{FeO}$ e $\mathrm{Fe}_{2} \mathrm{O}_{3}\left(\mathrm{Fe}_{3} \mathrm{O}_{4}\right)$ na estrutura cristalina e fase pura, sem presença de picos referentes a outras fases. Para a Pani pura, obtiveram-se picos entre $18^{\circ}-21^{\circ}(100)$ e entre $23^{\circ}-27^{\circ}(110)$, que correspondem aos planos da Pani cristalina. ${ }^{20,21} \mathrm{~A}$ presença de um pico intenso em $17,5^{\circ}$ e outros de menor intensidade em toda a extensão que o halo do polímero abrange no difratograma, são indicativos que o DBSA está presente como dopante nas cadeias da Pani, resultando em ramificações ordenadas em suas cadeias, o que a torna mais cristalina. ${ }^{22} \mathrm{O}$ DBSA atua junto à Pani organizando melhor sua estrutura, pois auxilia no processo de polimerização para que as cadeias sejam obtidas em unidades iguais reduzidas e oxidadas, o que leva a formação de uma rede reticulada estável e estrutura mais organizada. ${ }^{2,23}$ No compósito Pani-Mag há a presença marcante do polímero com predominância dos picos da Pani na região de 18 a $27^{\circ}$ com as indexações (100) e (110) respectivamente, e alguns picos da fase magnetita, como aqueles em $22^{\circ}$ (111), 29, $5^{\circ}$ (220), $32^{\circ}$ (311), $43^{\circ}(400)$ e $47^{\circ}(422)$, com menor intensidade, devido à elevada intensidade dos picos do polímero, o que indica que houve a interação sinérgica entre as fases e, provavelmente, a Pani está envolvendo as partículas de magnetita.

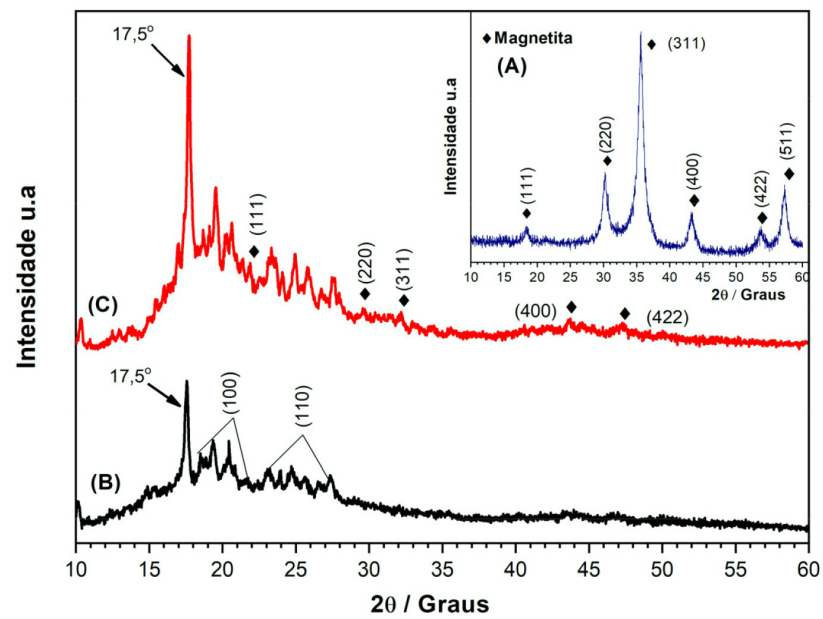

Figura 1. Difratogramas de raios X dos materiais (A) Magnetita, (B) Pani e (C) Pani-Mag

Pelas imagens de MEV apresentados na Figura 2, obteve-se homogeneidade no compósito com uma estrutura bem organizada e sem aglomerações, em que a magnetita encontra-se incorporada na estrutura Pani-DBSA e o polímero comporta-se como uma massa continua servindo de suporte para partículas de magnetita.

A Figura 3 apresenta os espectros de infravermelho da magnetita (A), Pani (B) e Pani-Mag (C), com as principais bandas e suas atribuições. Para a magnetita, destaca-se a banda dupla em $434 \mathrm{~cm}^{-1}$ e $585 \mathrm{~cm}^{-1}$, bem característica da fase, que pelos modos vibracionais de estiramento e torção, atribui-se à ligação $\mathrm{Fe}-\mathrm{O}^{24}$ e confirmam a presença de espinélio na estrutura de $\mathrm{Fe}_{3} \mathrm{O}_{4}$. No espectro da Pani pura, Figura 3B, são observadas duas bandas intensas na região de $1161 \mathrm{~cm}^{-1}$ a $1403 \mathrm{~cm}^{-1}$, que são atribuídas aos anéis benzênicos $(\mathrm{NH}-\mathrm{B}-\mathrm{NH})$ e quinoides $(\mathrm{N}=\mathrm{Q}=\mathrm{N})$. São características do estado de oxidação desejável, o sal esmeraldina, que se confirma também por bandas na região de $2928 \mathrm{~cm}^{-1}$ e $3142 \mathrm{~cm}^{-1}$, atribuídas à vibrações de estiramento $\mathrm{N}-\mathrm{H}$ dos grupos amino. Isso indica presença de grupos $-\mathrm{NH}_{2}$ nas unidades estruturais da Pani, com formação de cadeias estendidas e mais ordenadas..$^{20,21,23}$ Observa-se também a presença de bandas de absorções características ao DBSA na região de $587 \mathrm{~cm}^{-1} \mathrm{e} 1028 \mathrm{~cm}^{-1}$ confirmando sua interação com a 

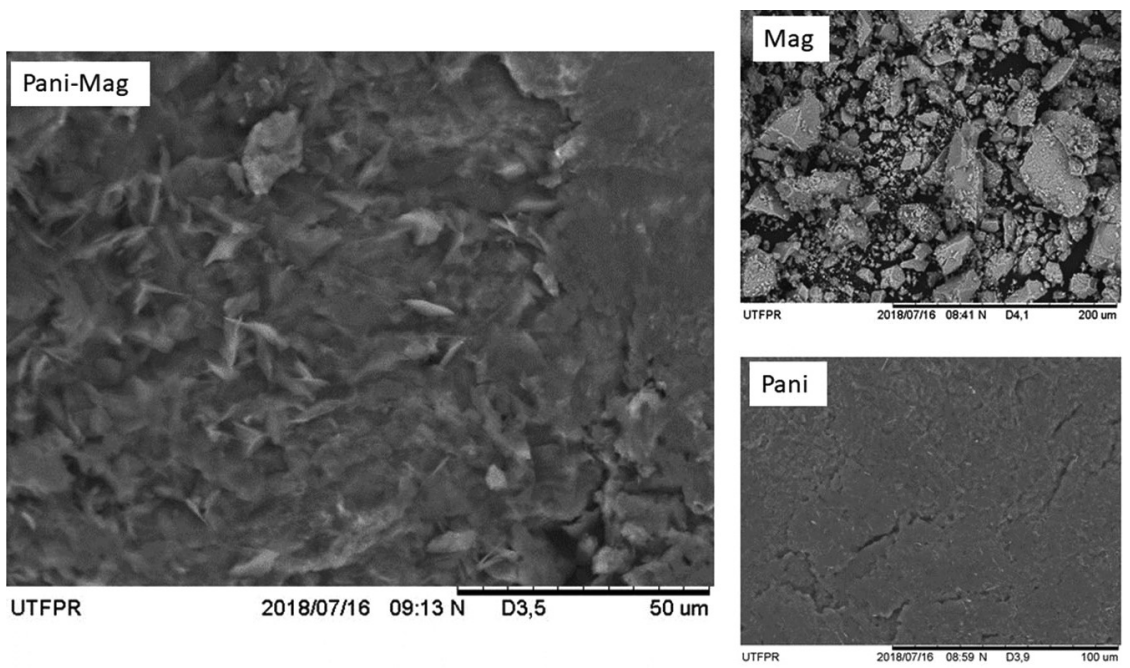

Figura 2. Imagens de Mev dos materiais Magnetita, Pani e Pani-Mag

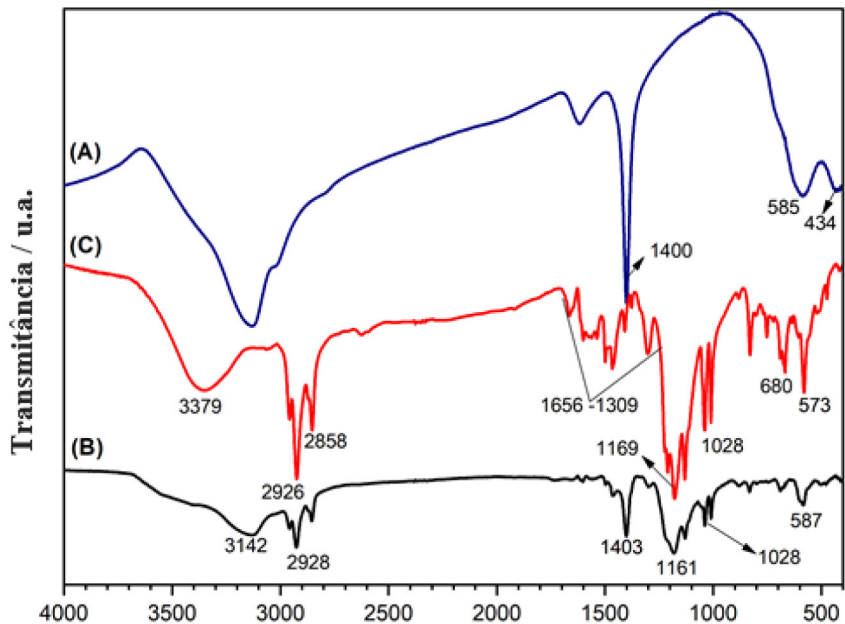

Número de onda $\mathrm{cm}^{-1}$

Figura 3. Espectro de Infravermelho para (A) Magnetita, (B) Pani e (C) Pani-Mag

Pani. ${ }^{23} \mathrm{O}$ espectro do compósito Pani-Mag, na Figura 3C, apresenta bandas intensas e bem definidas, com novos modos vibracionais em relação aos precursores. Principais bandas na região de $1028 \mathrm{~cm}^{-1}$ que se estendem até $1656 \mathrm{~cm}^{-1}$, características das interações de anéis benzênicos e quinoides do polímero, sugerindo também boa interação com o DBSA e maior organização estrutural. ${ }^{23,25} \mathrm{Em} 1169 \mathrm{~cm}^{-1}$ banda forte e intensa, e novos modos vibracionais nessa região sugerem novas ligações ou interações químicas nas estruturas poliméricas. As bandas características de magnetita ficaram encobertas pela fase orgânica, reiterando que houve interação do polímero com a fase inorgânica no compósito.

A Figura 4 apresenta as curvas de histerese da magnetita e do compósito Pani-Mag na temperatura ambiente. Através dessa curva, obtiveram-se dados de magnetização de saturação (Ms), que determina o valor máximo de magnetização. Para a magnetita obtevese valor de Ms máximo de 80,56 emu g-1 ${ }^{-1}$ estando compatível com valores da literatura, ${ }^{24,26,27} 64-70 \mathrm{emu} \mathrm{g}^{-1}, 76,5 \mathrm{emu} \mathrm{g}^{-1}, 64,7 \mathrm{emu} \mathrm{g}^{-1}$ respectivamente. O compósito Pani-Mag resultou em um valor máximo de Ms de 33,5 emu g ${ }^{-1}$, sendo que a literatura ${ }^{24,26}$ traz valores entre 41 e 62 emu g ${ }^{-1}$, e no caso da referência, ${ }^{28}$ que variou o teor de Fe em 30\%, 50\% e 70\% no compósito Pani-Magnetita-DBSA, obtendo-se resultados de magnetização também variável de 32, 56 e $78 \mathrm{emu} \mathrm{g}^{-1}$.

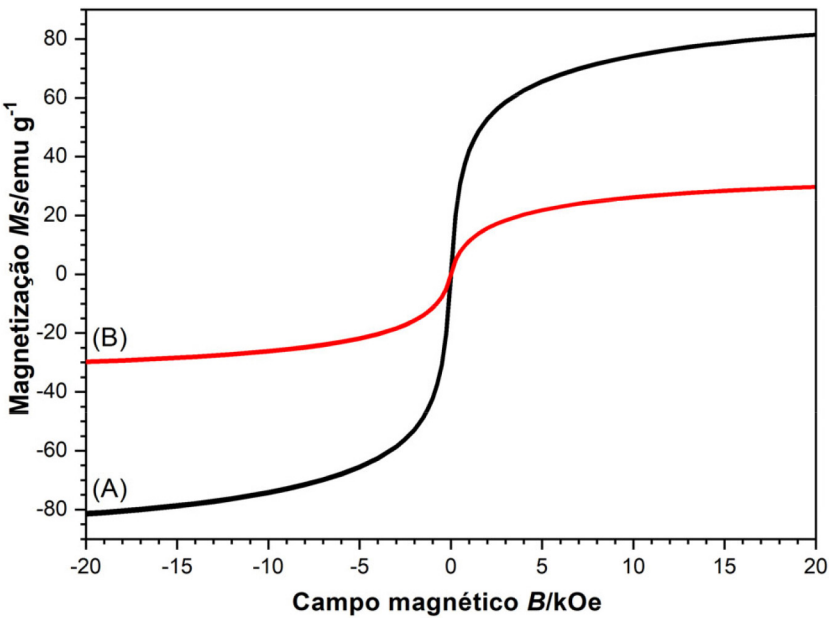

Figura 4. Curvas de magnetização das amostras (A) Magnetita e (B) Pani-Mag

Esse resultado é satisfatório para caracterizar o compósito Pani-Mag como magnético, que confere ao compósito melhora na estabilidade térmica, nas propriedades ópticas, eletroquímicas, magnéticas e mecânicas. Essas características o tornam promissor para aplicação em sensores e dispositivos eletroquímicos. ${ }^{27,29}$

Em geral, nos polímeros condutores não espera-se elevados valores de magnetização de saturação, pois a propriedade magnética depende da regularidade e da ordem estrutural das cadeias. Cadeias com maior linearidade e mais estendidas, permitem maior estabilidade de bipôlaron, o que leva a uma redução da contrubuição magnética. Por outro lado, quando as cadeias estao mais curtas, permitem mais pôlarons localizados, o que aumenta a magnetização. ${ }^{29}$

A magnetita tem facilidade de se aglomerar, o que resulta em magnetismo mais fraco e dispersabilidade. O tamanho de partícula e a quantidade de magnetita que será incorporada no polímero são cruciais para obter materiais com boas propriedades magnéticas. ${ }^{29}$

\section{Aspectos de sustentabilidade - Química Verde}

Na Figura 5, observa-se o gráfico de EV representando as cores verde, que comtemplam os critérios da métrica sustentável, e vermelho para o não cumprimento desses princípios. Quanto mais verde é o gráfico, maior é o índice de verdura e mais satisfatórios para princípios de sustentabilidade. O IPE obtido foi de $65 \%$ em escala que varia de 0 a 100, o que é considerado aceitável com bons indícios de uma síntese sustentável, apesar de ter sido utilizado 
reagentes que representam risco potencial para a saúde e ambiente como a anilina e o DBSA.

Destaca-se que atingiram nível máximo satisfatórios, os princípios: P2-economia atômica, P6-planificação para eficácia energética, P7matérias primas renováveis, $\mathrm{P} 8$-derivatizações e $\mathrm{P} 9$-catalisadores. Não se mostrou satisfatória para os critérios P3-sínteses perigosas, e P12mais segura na prevenção de acidentes. Alguns princípios atingiram nível intermediário, como os critérios P1-prevenção, P5-solventes e substâncias auxiliares mais seguras, e P10-degradação, em que se obteve um nível intermediário para princípios sustentáveis. É possível melhorar os parâmetros necessários para atingir a verdura máxima, dentro das possibilidades e otimizando o processo.

Essa métrica de análise de dados reforça a importância de realizar estudos das condições de síntese a fim de torná-la mais sustentável, utilizando menores quantidades de reagentes, tendo maior economia e menores quantidades de resíduos. Houve a simplificação de etapas e processos que envolviam riscos físicos, para o meio ambiente e para a saúde e foi obtido material compósito com características satisfatórias para aplicações tecnológicas.

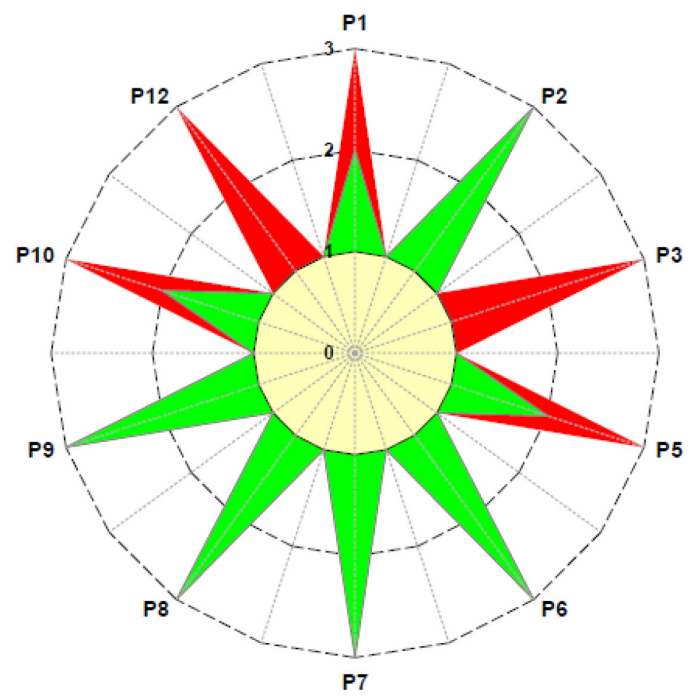

Figura 5. EV para síntese do compósito Pani-Mag

\section{Caracterização eletroquímica e ensaios como sensor para o ácido ascórbico}

Com o intuito de analisar um potencial de aplicabilidade desse material compósito como sensor com molécula sonda de Ácido Ascórbico (AA), foi realizada a caracterização eletroquímica descrita a seguir.

A Figura 6 apresenta o perfil eletroquímico, caracterizado por voltametria cíclica, da Pani e do compósito. O voltamograma da Pani pura apresentou, dois pares de oxidação: identificados como $1($ em $0,26 \mathrm{~V})$ e $2($ em $0,76 \mathrm{~V})$ que correspondem aos processos de oxidação do estado leucoesmeraldina-esmeraldina (polarônica) e esmeraldina-pernigranilina (bipolarônica). E dois pares de reduções: identificados como 2' (em 0,68 V) e 1' (em 0,09 V), correspondentes ao processo de redução de pernigranilina-esmeraldina e esmeraldinaleucoesmeraldina, confirmando a presença da Pani na forma condutora. ${ }^{16}$

Já o conjunto de picos em 3 e 3' correspondem a efeitos de degradação do polímero e formação de intermediários como quinonas e hidroquinonas, quebra de ligações da cadeia polimérica e formação de ligações intercadeias na etapa de síntese. Esses intermediários são indesejados, visando-se a uma aplicação em sensores, pois indicam que não houve estabilidade nos processos redox. ${ }^{16,29}$
No voltamograma do compósito Pani-Mag, observam-se dois conjuntos de oxidação/redução, $1($ em $0,33 \mathrm{~V})$ e $2($ em $0,65 \mathrm{~V})$ e o segundo, 2' (em 0,31 V) e 1' (em 0,09 V), respectivamente, sugerindo a presença de Pani na forma condutora, uma vez que são atribuídos às transições características dessa fase. Observa-se melhor estabilidade de ciclagens pelas intensidades da densidade de corrente que foi menor em relação à Pani, e pelo fato de não aparecerem picos intermediários de degradação, que são atribuídos ao sinergismo entre as partículas magnéticas e o polímero.

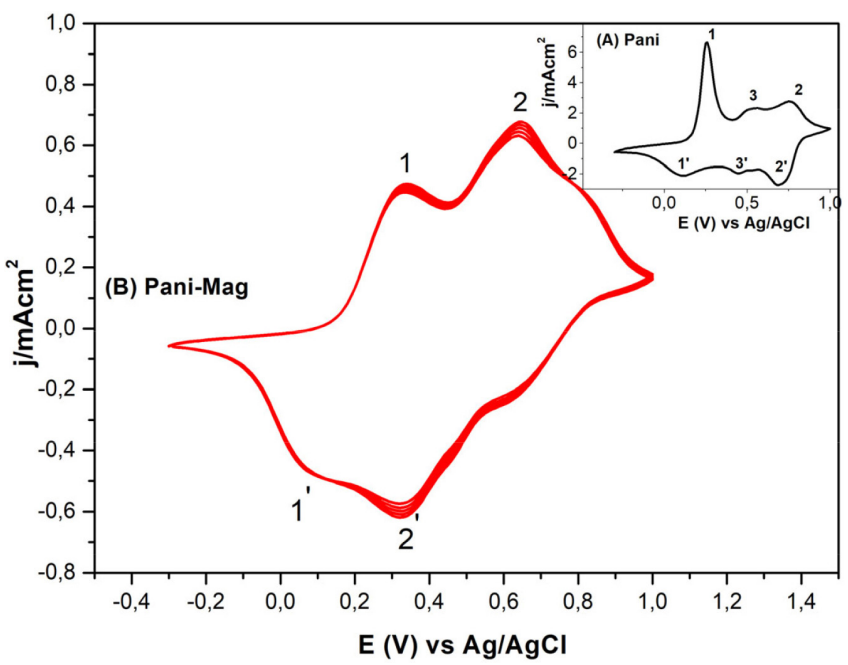

Figura 6. VC do compósito Pani-Mag em eletrodo de Carbono vítreo em $\mathrm{H}_{2} \mathrm{SO}_{4} 0,5 \mathrm{~mol} \mathrm{~L}^{-1}$

Os resultados apresentados mostram que o compósito sintetizado tem as fases polimérica e partículas magnéticas em sinergismo, contribuindo para melhorar as propriedades eletroquímicas da Pani, mantendo as propriedades magnéticas da magnetita, conforme as análises de magnetização.

As características apresentadas são importantes em um sensor eletroquímico, que deve ter resposta de análise rápida, estabilidade eletroquímica, fácil manuseio e preparo do eletrodo de trabalho. Além disso, o compósito tem possibilidade de melhorar o desempenho do eletrodo como sensor. Através da transferência de elétrons por oxidação e redução, melhora a área superficial e reduz a possibilidade de contaminações do eletrodo. ${ }^{30}$

Considerando que a estabilidade observada pela boa interação entre as fases e que as características magnéticas e eletroquímicas da Pani foram melhoradas devido à presença da magnetita, foi proposto utilizar este material como um sensor eletroquímico de ácido ascórbico. Não foi encontrada na literatura a aplicação de material semelhante para essa finalidade, apenas exemplos com eletrodo modificado de pasta de carbono com magnetita ${ }^{13}$ e eletrodo modificado com Carbono e Nafion. ${ }^{12}$ Estudos preliminares aqui apresentados apontam para um sistema promissor.

Para evidenciar o potencial de aplicabilidade do compósito, realizaram-se testes eletroquímicos para sensores, que determinassem o perfil de oxidação do ácido ascórbico, à medida que sua concentração aumentava na cela eletroquímica. Testes foram realizados utilizandose o compósito Pani-Mag em suspensão gotejados na superfície do eletrodo de trabalho, obtendo-se como melhores condições em solução tampão de fosfato de potássio $0,1 \mathrm{~mol} \mathrm{~L}^{-1} \mathrm{em} \mathrm{pH} \mathrm{4,0} \mathrm{e}$ intervalo de potencial de $-0,2$ a $1,0 \mathrm{~V}$ e escala de corrente de $1 \mu \mathrm{A}$ e velocidade de varredura de $50 \mathrm{mV} \mathrm{s}^{-1}$. Solução padrão de AA na concentração de $0,1 \mathrm{~mol} \mathrm{~L}^{-1}$ foi adicionada em 10 alíquotas sucessivas de $100 \mu \mathrm{L}$ cada, com posterior leitura do voltamograma cíclico a fim de acompanhar o perfil de oxidação, em função da concentração 
do analito que vai aumentando na célula eletroquímica. Um branco foi realizado apenas com o eletrodo modificado na superficie do eletrodo, sem adição de alíquotas de AA em solução, em que houve estabilização no perfil após ciclagens. Somente após adicionar o analito que gradativamente foi modificando o perfil eletroquímico, conforme descrito na figura a seguir.

$\mathrm{Na}$ Figura 7, em (A) observa-se o comportamento redox do AA, sendo que conforme aumenta a concentração do analito, aumenta a corrente de pico, evidenciado em (B). Esse aumento da corrente é devido à taxa de transferência de elétrons na superfície do eletrodo de carbono vítreo que se encontra modificada com o compósito Pani-Mag. ${ }^{12} \mathrm{Na}$ faixa de potencial de trabalho é o único conjunto redox detectado.
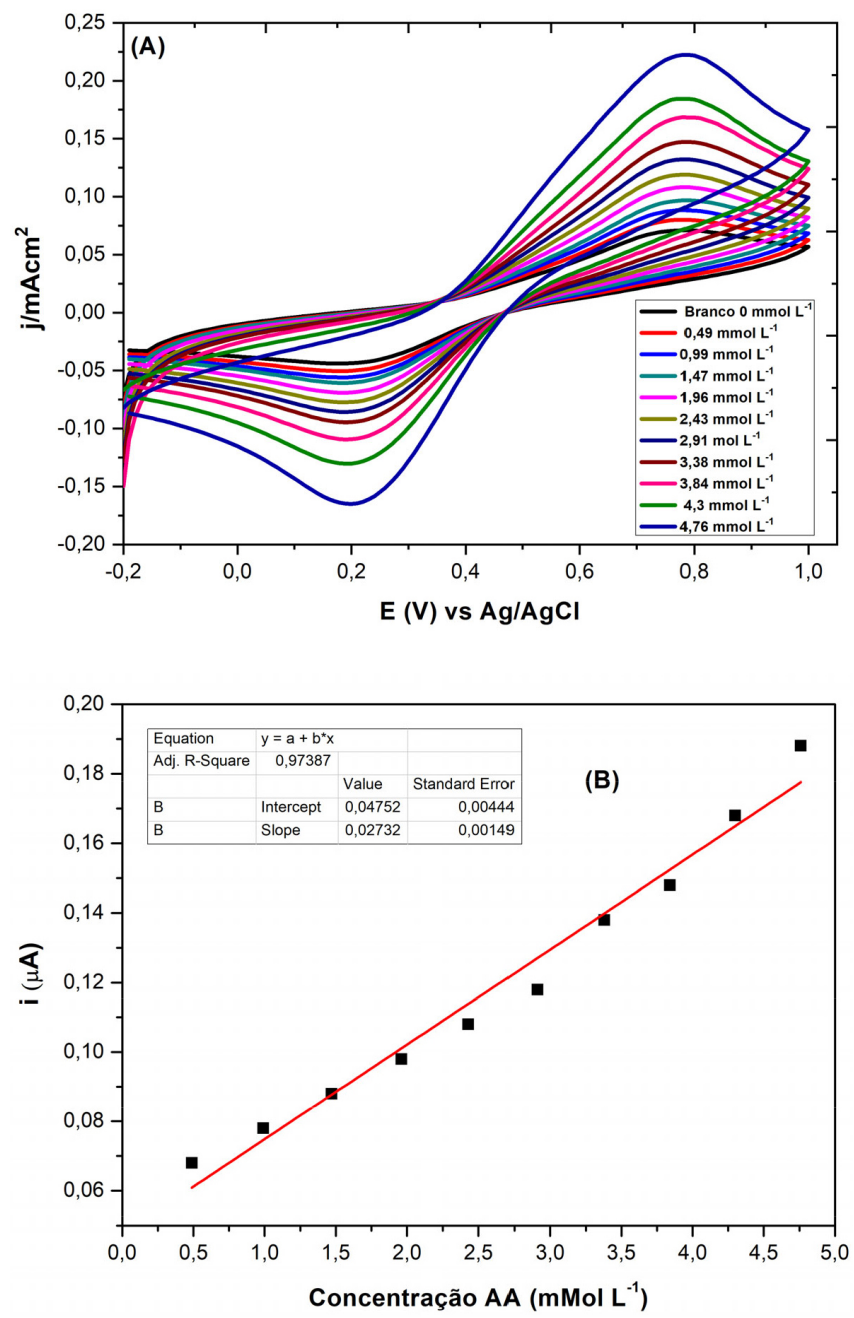

Figura 7. (A) Perfil de oxidação de AA com eletrodo modificado com o compósito Pani-Mag, (B) concentração em função da corrente

A presenca do óxido no compósito Pani-Mag se mostrou mais sensível, mais estável eletroquimicamente e com melhores respostas eletroquímicas ao se comparar com eletrodo modificado apenas com Pani. Vale ressaltar que satisfaz aos objetivos, pois a intenção era qualificação do material eletroativo.

Esse resultado mostra que o compósito Pani-Mag é promissor para aplicação como sensor de AA. Testes analíticos de limites de detecção e quantificação serão realizados para determinar esses parâmetros em trabalhos futuros. No entanto, pela resposta obtida no teste de voltametria, observou-se que há resposta para sensor de detecção do analito ácido ascórbico em solução, sendo essa uma aplicação de interesse biológico e tecnológico.

\section{CONCLUSÕES}

Foi obtido um material compósito entre Pani e magnetita através de síntese química que se mostrou viável em termos técnico e econômico e, portanto, promissora para desenvolvimento de novos materiais de interesse tecnológico. Os parâmetros métricos de química verde foram atendidos de maneira satisfatória, com foco em sínteses mais sustentáveis do ponto de vista ambiental, econômico e para a saúde.

O compósito obtido apresentou características químicas e eletroquímicas que somaram positivamente, para os efeitos eletroquímicos e de magnetização, considerando o polímero e o óxido isoladamente. Possui potencial para ser aplicado em dispositivos eletroquímicos, nesse caso, os sensores. Houve boa resposta para sensor de AA, que ressalta ainda mais a importância desse estudo, uma vez que pode ser uma metodologia para sua determinação em amostras analíticas.

\section{AGRADECIMENTOS}

Os autores agradecem às agências de fomento: CAPES, CNPq, FINEP e Fundação Araucária. À Central de Análises da UTFPR e ao Laboratório multiusuário de caracterização magnética da UFSC Campus Florianópolis, em especial ao G. Candiotto.

\section{REFERÊNCIAS}

1. Reddy, K. R.; Hemavathi, B.; Balakrisma, G. R.; Raghu, A. V.; Naveen, S.; Shankar, M. V.; Polymer composites with funcionalized nanoparticles, 2019, 11.

2. Vargas, V. M. M.; Dalmolin, C.; Pezinn, S. H.; Oliveira, M. M.; Zamora, P. P.; Quim. Nova 2018, 41, 315.

3. Shalini, A.; Nishanthi, R.; Palani, P.; Jaisankar, V.; Mater. Today: Proc. 2016, 3, 1633.

4. Fleaca, T. C.; Dumitrache, F.; Morjan, I.; Niculescu, A. M.; Sandu, I.; Ilie, A.; Stamantin, I.; Iordache, A.; Vasile, E.; Prodan, F.; Appl. Surf. Sci. 2016, 374, 213.

5. Kango, S.; Kalia, S.; Celli, A.; Njuguna, J.; Habibi, Y.; Kumar, R.; Prog. Polym. Sci. 2013, 38, 1232.

6. Mallakpour, S.; Madani, M.; Prog. Org. Coat. 2015, 85, 194.

7. Ballarin, B.; Boanini, E.; Montalto, L.; Mengucci, P.; Nanni, D.; Parise, C.; Ragazinni, I.; Rinaldi, D.; Sangiorgi, N.; Sanson, A.; Cassani, M. C.; Electrochim. Acta 2019, 322.

8. Prasankumar, T.; Wiston, B. R.; Gautam, C. R.; Ilangovan, R.; Jse, S. P.; J. Alloys Compd. 2018, 757, 466.

9. Misurovic, J.; Mojovic, M.; marjanovic, B. Synth. Met. 2019, 257, 116.

10. Batool, R.; Akhtar, M. A.; Hayat, A.; Han, D.; Niu, L.; Ahmad, M. A.; Nawaz, M. H.; Microchim. Acta 2019, 186, 267.

11. Jaime, J.; Rangel, G.; Bonilla, A. M.; Mayoral, A.; Herrasti, P.; Sensors Actuators, B 2017, 238, 693.

12. Araújo, K. R. O.; Dissertação de Mestrado, Universidade Federal de Goiás, Brasil, 2017.

13. Nascimento, D. C. F.; Dissertação de Mestrado, Universidade Federal de Alagoas, Brasil, 2019.

14. Kurlyandskaya, G. V.; J. Phys. Chem. Solids 2007, 68, 1527.

15. Alves, K. G. B.; Tese de doutorado, Universidade Federal de Pernambuco, Brasil, 2010.

16. Radhakrishnan, S.; Cunanan. J.; Bhagat, S. M.; Aphesteguy, J. C.; Jacobo, S. E.; J. Appl. Polym. Sci. 2011, 122, 1510.

17. Ribeiro, M. G. T. C.; Yunes, S. F.; Machado, A. S. C.; J. Chem. Educ. 2014, 91, 1901 .

18. Magela, W. F.; Resumos do XVII ENEQ, Encontro Nacional de Estudantes de Química, Florianópolis, Brasil, 2016. 
19. Cornell, R. M.; The iron oxides: structure, properties, reactions, occurrences and uses, Wiley-VCH Verlag: Germany, 2003, cap. 6 e 7.

20. Gu, H.; Huang, Y.; Zhang, X.; Wang, X.; Zhu, J.; Shao, L.; Young, D. P.; Wei, S.; Guo, Z.; Polymer 2012, 53, 801.

21. Basavaiah, K.; Kumar, Y. P.; Rao, A. V. R.; Appl. Nanosci. 2013, 3, 409.

22. Han, D.; Chu, Y.; Yang, L.; Lv, Z.; Colloids Surf. 2005, 259, 179

23. Kumar, V.; Yokozeki, T.; Goto, T.; Takahashi, T.; Polymer 2016, 86, 129.

24. Araujo, A. C. V.; Oliveira, R. J.; Alves, S. A.; Rodrigues, A. R.; Machado, F. L. A.; Cabral, F. A. O.; Azevedo, W. M.; Synth. Met. 2010, 160,685 .

25. Aliramaji, S.; Zamanian, A.; Sohrabijam, Z.; Procedia Mater. Sci. 2015, $11,265$.
26. Zang, Y. J.; Lin, Y. W.; Chang, C. C.; Wu, T. M.; Synth. Met. 2011, 161, 937.

27. Diniz, V. C. S.; Dantas, B. B.; Figueireo, A. R.; Cornejo, D. R.; Costa, A. C. F. M.; Cerâmica 2015, 61, 298.

28. Xue, W.; Qiu, H.; Fang, K.; Li, J.; Zhao, J.; Li, M.; Synth. Met. 2006, $156,833$.

29. Marchesi, L. F. Q. P.; Tese de Doutorado, Universidade Federal de São Carlos, Brasil, 2010.

30. Iqbal, S.; Ahmad, S.; J. Ind. Eng. Chem. 2018, 60, 53. 